\title{
The fraction of RNA that folds into the correct branched secondary structure determines hepatitis delta virus type 3 RNA editing levels
}

\author{
SARAH D. LINNSTAEDT, ${ }^{1}$ WOJCIECH K. KASPRZAK, ${ }^{2}$ BRUCE A. SHAPIRO, ${ }^{3}$ and JOHN L. CASEY ${ }^{1}$ \\ ${ }^{1}$ Department of Microbiology and Immunology, Georgetown University Medical Center, Washington, DC 20007, USA \\ ${ }^{2}$ Basic Research Program, SAIC-Frederick, Inc., National Cancer Institute at Frederick (NCI-Frederick), Frederick, Maryland 21702, USA \\ ${ }^{3}$ Center for Cancer Research Nanobiology Program, National Cancer Institute at Frederick (NCI-Frederick), Frederick, Maryland 21702, USA
}

\begin{abstract}
RNA editing by the host RNA adenosine deaminase ADAR1 at the amber/W site of hepatitis delta virus RNA plays a central role in the viral replication cycle by affecting the balance between viral RNA synthesis and packaging. Previously, we found that HDV genotype III (HDV-3) RNA can form two secondary structures following transcription: an unbranched rod structure, which is characteristic of $\mathrm{HDV}$, and a metastable branched structure that serves as the substrate for editing. The unstable nature of the branched editing substrate structure raised the possibility that structural dynamics of the RNA following transcription could determine the rate at which editing occurs. Here, editing and its control are examined in two HDV-3 isolates, from Peru and Ecuador. Analysis of editing in vitro by ADAR1 indicated that the branched structure formed by RNA derived from the Peruvian isolate is edited more efficiently than that from the Ecuadorian isolate. In contrast, in the context of replication, Peruvian RNA is edited less efficiently than RNA containing Ecuadorian sequences. Computational analyses of RNA folding using the massively parallel genetic algorithm (MPGAfold) indicated that the Peruvian RNA is less likely to form the branched structure required for editing than the Ecuadorian isolate. This difference was confirmed by in vitro transcription of these RNAs. Overall, our data indicate that HDV-3 controls RNA editing levels via (1) the fraction of the RNA that folds, during transcription, into the metastable branched structure required for editing and (2) the efficiency with which ADAR1 edits this branched substrate RNA.
\end{abstract}

Keywords: RNA editing; RNA structural dynamics; hepatitis delta virus; ADAR1; RNA folding

\section{INTRODUCTION}

The circular 1.7-kb HDV RNA genome of the hepatitis delta virus (HDV), the smallest known to infect man, produces just one protein, hepatitis delta antigen (HDAg), which is encoded by less than half of the genome (Wang et al. 1986). This limited coding capacity is compensated by structural features in both the genomic and antigenomic RNAs and by host functions. One example of this compensation is editing of a fraction of the HDV antigenome at the amber/W site by the host RNA adenosine deaminase ADAR1 (Casey 2006), which acts on adenosines in certain RNA secondary structures in viral and host mRNAs and

Reprint requests to: John L. Casey, Department of Microbiology and Immunology, Georgetown University Medical Center, 3900 Reservoir Road, NW Washington, DC 20007, USA; e-mail: caseyj@georgetown.edu; fax: (202) 687-1800.

Article published online ahead of print. Article and publication date are at http://www.rnajournal.org/cgi/doi/10.1261/rna.1504009.
miRNAs. Editing at the HDV amber/W site changes the amber stop codon of the short form of HDAg to a tryptophan codon. As a result, the virus produces two forms of HDAg that play critical roles in the replication cycle (Kuo et al. 1989; Chang et al. 1991; Casey et al. 1992; Jayan and Casey 2002b).

Control of the rate and extent of editing is essential for HDV because the form of HDAg produced as a consequence of editing-HDAg-L_-inhibits viral RNA synthesis but is also a limiting factor for virus particle formation (Chao et al. 1991; Jayan and Casey 2002a; Sato et al. 2004; Jayan and Casey 2005). Editing levels of a number of cellular and viral RNAs vary considerably, not only among different sites, but for the same site in different tissues, stages of development, and pathological settings (Bass 2002; Keegan et al. 2004; Maas et al. 2006; Gandy et al. 2007; Cenci et al. 2008; Riedmann et al. 2008). As yet, the factors that determine these variations are not well understood. For HDV genotype I (HDV-1), editing is down-modulated 
in part by HDAg-S (Polson et al. 1998; Sato et al. 2004), most likely by binding to the RNA. However, we previously observed that HDAg-S is not an effective inhibitor of amber/W site editing for HDV-3, which differs from HDV-1 by $\sim 40 \%$ in nucleic acid sequence (Cheng et al. 2003), thus indicating that an alternative mechanism must exist for the regulation of editing for this genotype. Unlike HDV-1, amber/W site editing in HDV-3 requires a branched secondary structure comprised of a central base-paired region containing the amber/W site flanked by two $\sim 25$-base-pair stem-loops, designated SL1 and SL2 (Casey 2002; Linnstaedt et al. 2006). This structure is energetically less stable than the unbranched rod structure that is characteristic of HDV RNA and is required for RNA replication (Linnstaedt et al. 2006). Because only the metastable branched structure can be edited, the distribution of the antigenomic RNA between the two structures following transcription could be an important determinant of the amount of editing that occurs during viral RNA replication.

Here, we compare RNA folding dynamics (in silico and in vitro) and editing (in vitro and in vivo) in two HDV-3 isolates, one from Peru (Casey et al. 1993; Casey 2002), the other from Ecuador (Manock et al. 2000; Linnstaedt et al. 2006). We find that HDV-3 controls RNA editing levels via (1) the fraction of the RNA that folds, following transcription, into the metastable branched structure required for editing and (2) the efficiency with which ADAR1 edits this branched substrate RNA. Overall, we conclude that HDV-3 controls editing levels, in part, by limiting the fraction of RNA that forms the branched editing substrate following transcription.

\section{RESULTS}

\section{The branched structures of two HDV-3 isolates are edited with different efficiencies by ADAR1 in vitro}

We examined amber/W site editing and its control in HDV-3 using two temporally and geographically distinct isolates, one from Peru and one from Ecuador (Casey et al. 1993; Manock et al. 2000). These isolates differ at 17nucleotide (nt) positions in the $\sim 300$-nt region required for editing. Previously, we used a 320-nt miniaturized version of the Ecuadorian isolate RNA, referred to here as $\mathrm{mE}$, that can form both the branched structure required for editing at the amber/W site and the unbranched rod structure characteristic of HDV RNA (Linnstaedt et al. 2006). Stem-flip (SF) mutations were introduced in the stems of stem-loops SL1 and SL2 (Fig. 1A) of the branched structure to create the miniaturized RNA mE-SF (previously referred to as MD-III-2SF; Linnstaedt et al. 2006), which forms only the branched structure and is efficiently edited (Linnstaedt et al. 2006).
In order to determine whether the sequence differences between the Peruvian and Ecuadorian isolates affect the editing efficiency of the branched structure, we created the miniaturized RNA mP-SF, which is derived from the HDV Peruvian isolate and is analogous to $\mathrm{mE}-\mathrm{SF}$ in that it efficiently forms the branched structure in vitro. In vitrotranscribed, gel-purified mP-SF and mE-SF RNAs were incubated with nuclear extract obtained from cells transfected with an ADAR1 expression vector (Wong et al. 2003), and the extent of editing at the amber/W site was determined using a previously described RT-PCR and Sty I digestion assay (Linnstaedt et al. 2006). We observed that mP-SF RNA was edited threefold more efficiently than $\mathrm{mE}-$ SF RNA (Fig. 1B), indicating that some or all of the $17 \mathrm{nt}$ that differ between the two isolates affect the efficiency with which these branched RNAs are edited.

Several studies of the secondary structure requirements for editing of different substrates have shown that the

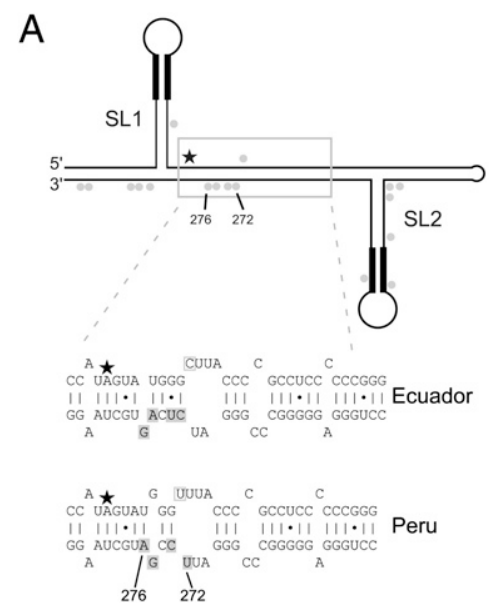

B
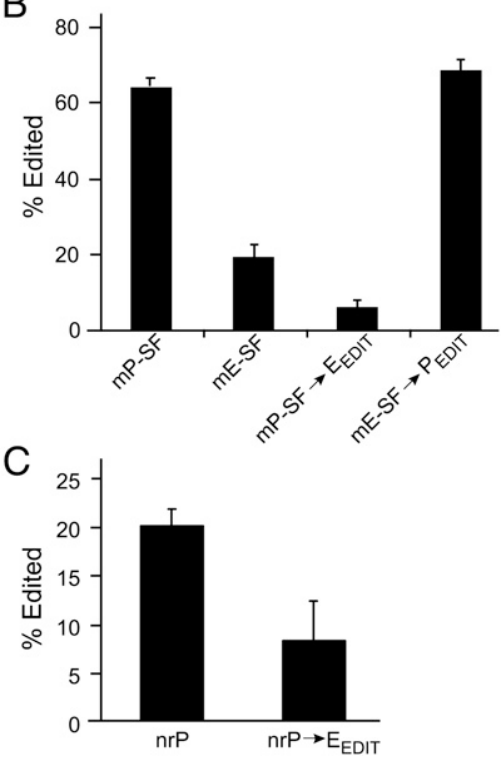

FIGURE 1. (Legend on next page) 
efficiency of editing is particularly dependent on base pairing 3' of the editing site (Polson and Bass 1994; Lehmann and Bass 1999; Aruscavage and Bass 2000; Ohman et al. 2000; Bhalla et al. 2004; Dawson et al. 2004). Five of the $17 \mathrm{nt}$ that differ between the Ecuadorian and Peruvian miniaturized RNAs are in the base-paired region $3^{\prime}$ of the amber/W site (Fig. 1A). Four of these $5 \mathrm{nt}$ are grouped between positions 272 and 276 and affect the predicted secondary structure in this region (Fig. 1A). In order to examine the role of these sequence differences in the editing efficiencies of the $\mathrm{mP}-\mathrm{SF}$ and $\mathrm{mE}-\mathrm{SF}$ branched RNAs, we created RNAs $\mathrm{mP}-\mathrm{SF} \rightarrow \mathrm{E}_{\mathrm{EDIT}}$ and $\mathrm{mE}-\mathrm{SF} \rightarrow$ $\mathrm{P}_{\mathrm{EDIT}}$, in which these $4 \mathrm{nt}$ were exchanged, and analyzed editing by ADAR1 in vitro. We found that substituting these four variant nucleotides in $\mathrm{mP}-\mathrm{SF}$ RNA with those from the Ecuadorian isolate reduced editing by almost 10fold (Fig. 1B, mP-SF $\rightarrow \mathrm{E}_{\mathrm{EDIT}}$ ); the reverse substitution (Fig. $1 \mathrm{~B}, \mathrm{mE}-\mathrm{SF} \rightarrow \mathrm{P}_{\mathrm{EDIT}}$ ) raised the level of editing to that of the branched Peruvian RNA. We also examined whether the cluster of $3 \mathrm{nt}$ near the base of SL2 affects editing of branched RNAs. No difference in editing was detected when these three bases were exchanged between $\mathrm{mE}-\mathrm{SF}$ and mP-SF (data not shown). Thus, the four nucleotide variations at positions $272-276$ play a critical role in determining the efficiency with which the branched $\mathrm{mE}$ and $\mathrm{mP}$ RNAs are edited, and likely account for the majority of the difference in editing efficiency between them. That the editing level of $\left(\mathrm{mP}-\mathrm{SF} \rightarrow \mathrm{E}_{\mathrm{EDIT}}\right)$ is lower than $\mathrm{mE}-\mathrm{SF}$ suggests that additional sequence differences between $\mathrm{mE}-\mathrm{SF}$ and $\mathrm{mP}-\mathrm{SF}$, such as the fifth nucleotide in

FIGURE 1. HDV RNAs from Peruvian and Ecuadorian isolates are edited with different efficiencies. (A) Schematic diagram of the branched RNA secondary structure formed by miniaturized RNAs $\mathrm{mE}-\mathrm{SF}$ and $\mathrm{mP}-\mathrm{SF}$ derived from the Ecuadorian and Peruvian HDV-3 isolates, respectively. The star denotes the amber/W editing site. Heavy lines indicate the location of the stem-flip (SF) mutations that stabilize stem-loops SL1 and SL2 in this branched structure. The $17 \mathrm{nt}$ positions that differ between the Peruvian and Ecuadorian miniaturized RNAs are indicated by gray circles. The region around the editing site is enlarged, showing the sequence and predicted secondary structure of the Ecuadorian and Peruvian isolate RNAs. WatsonCrick base pairs are indicated by vertical lines, $\mathrm{G}-\mathrm{U}$ wobble pairs by black dots. The sequence differences between the two RNAs are shaded or outlined in gray. $(B)$ Editing in vitro by ADAR1 of miniaturized branched Peruvian and Ecuadorian RNAs. RNAs were incubated with nuclear extracts containing ADAR1; percent editing of the RNA at the amber/W site was determined by restriction digestion of RT/PCR products, as described (Linnstaedt et al. 2006). Values shown are the average of four independent experiments. The four variant positions between 272 and 276 (shaded gray in $A$ ) were exchanged between $\mathrm{mP}-\mathrm{SF}$ and $\mathrm{mE}-\mathrm{SF}$ to generate $\mathrm{mP}-\mathrm{SF} \rightarrow \mathrm{E}_{\mathrm{EDIT}}$ and $\mathrm{mE}-\mathrm{SF} \rightarrow \mathrm{P}_{\mathrm{EDIT}}$. $(C)$ Editing of nonreplicating RNAs in transfected cells. Huh-7 cells were transfected with expression constructs for nonreplicating RNAs Pnr and Pnr $\rightarrow \mathrm{E}_{\mathrm{EDIT}}$ (described in the text). The former contains Peruvian HDV sequences, the latter contains the four sequences of the Ecuadorian variant between positions 272 and 276. RNAs were harvested $2 \mathrm{~d}$ post-transfection and analyzed for amber/W site editing, as described previously (Cheng et al. 2003). the cluster, at position 117, might also contribute to variations in substrate editing efficiency.

We also compared the activities of the Peruvian and Ecuadorian editing sites in transfected cells. For this analysis we used expression constructs for nonreplicating RNAs from which SL1 and SL2 have been removed (Cheng et al. 2003). These deletions are predicted to favor the formation of the central base-paired region containing the amber/W site in the branched editing conformation over the formation of the structure around the amber/W site that occurs in the native unbranched rod structure and, consistent with this prediction, were shown to increase editing in transfected cells (Cheng et al. 2003). Replacement of the $4 \mathrm{nt}$ in the nonreplicating Peruvian RNA, Pnr, with Ecuadorian sequences, Pnr $\rightarrow \mathrm{E}_{\mathrm{EDIT}}$, resulted in a threefold reduction in editing (Fig. 1C). Thus, both in vitro with ADAR1 and in cells, the Peruvian HDV amber/W site is a more efficient editing site than the Ecuadorian.

\section{In the context of viral replication, editing levels for the Ecuadorian isolate are higher than for the Peruvian isolate}

The results shown in Figure 1 demonstrate that, when the RNA is restricted to the branched conformation by SF mutations, ADAR1 edits the Peruvian amber/W site more efficiently than the Ecuadorian amber/W site. In order to determine whether Ecuadorian and Peruvian HDV RNAs also exhibit these distinct editing phenotypes during viral RNA replication, we analyzed editing following transfection of Huh-7 cells with replication-competent expression constructs. For the Peruvian RNA, we used a previously described construct, pHDV•III(+) (referred to here as fl$\mathrm{P})$, that consists of 1.2 copies of the genome; transfection of this construct produces full-length replicating HDV RNA that is edited at the amber/W site (Casey 2002). For the Ecuadorian RNA, we created a chimeric construct, fl-E, which includes the sequences from the coding and noncoding segments of the Ecuadorian isolate that make up $\mathrm{mE}$ (Fig. 2A). Huh-7 cells were transfected with these constructs, and total cellular RNAs harvested on days 6, 9, and 12 were analyzed for editing at the amber/W site. Surprisingly, and contrary to what was expected based on the results obtained for branched RNA editing in vitro, fl-P RNA was edited at lower levels than fl-E. This relationship was observed consistently between 6 and $12 \mathrm{~d}$ posttransfection (Fig. 2B).

To ensure that the higher editing level observed for the Ecuadorian isolate was not an artifact of using a chimeric construct derived from both Peruvian and Ecuadorian RNAs, we examined editing levels of virion RNA in the serum samples from which the cDNA clones were derived. We observed that, consistent with the results obtained for the replicating RNA in transfected cells, the viral RNA in the serum of the Peruvian isolate was edited at a lower 


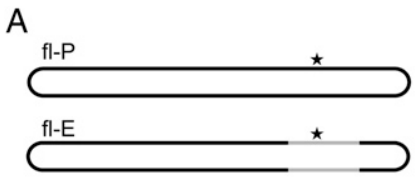

C

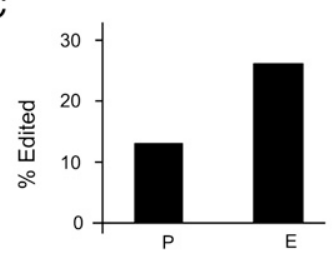

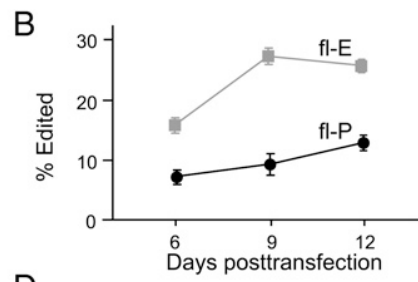

D

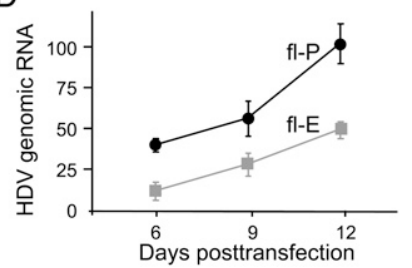

FIGURE 2. Comparison of editing in replicating RNAs. (A) Schematic diagram of replication-competent full-length RNAs fl-P and flE used to compare editing of replicating Peruvian and Ecuadorian HDV RNAs. The full-length Peruvian RNA, fl-P, is generated from a 1.2-length cDNA expression construct following transfection of Huh7 cells (Casey and Gerin 1998). For the Ecuadorian RNA we created a chimeric construct to generate a full-length RNA, fl-E, that contains 15 of the Ecuadorian sequence differences in the branched structure that forms the substrate for editing (Fig. 1); the remainder consists of Peruvian sequences. Black indicates Peruvian sequences, gray indicates sequences (positions on replicating RNA) derived from the Ecuadorian isolate. (B) Huh-7 cells were transfected with expression constructs for fl-P and fl-E RNAs. Amber/W site editing was assayed on RNA isolated 6, 9, and $12 \mathrm{~d}$ post-transfection. Percent editing was determined as in Figure 1. Results are the average of three independent experiments. $(C)$ Analysis of editing in HDV RNA isolated from patient sera. (D) HDV RNA levels in Huh-7 cells transfected with either fl-P (black circles) or fl-E (gray squares) antigenomic RNA expression constructs. RNAs were harvested on the indicated days post-transfection and analyzed by blot hybridization for levels of HDV genomic RNA. Three independent transfections were analyzed; error bars indicate standard deviations.

level than that of the Ecuadorian isolate (Fig. 2C). From these results, it is clear that, while the branched RNA derived from the Peruvian isolate is edited more efficiently, the Ecuadorian RNA is edited to a higher level during replication.

Editing is responsible for the production of HDAg-L, which inhibits HDV RNA replication. We and others have previously shown that variations in editing levels affect the amount of HDV RNA replication (Casey 2002; Jayan and Casey 2002a; Sato et al. 2004; Jayan and Casey 2005). In those studies, editing levels were varied by alterations of ADAR expression levels and/or the sequence around the editing site. Interventions that increased levels of editing were correlated with decreased levels of HDV RNA replication. Similarly, the higher levels of amber/W site editing in cells replicating fl-E, as compared with fl-P (Fig. 2B), were correlated with a lower level of replication for fl-E RNA than fl-P RNA (Fig. 2D). This result is consistent with the effects of a higher level of HDAg-L expression in cells replicating fl-E RNA, and indicates that the level of editing can have a significant effect on viral replication.

\section{The MPGAfold algorithm indicates that Peruvian RNA has a decreased tendency to form the branched structure required for editing}

We considered that the different relative levels of editing exhibited by the Ecuadorian and Peruvian RNAs in Figures 1 and 2 could be due to differences in the folding dynamics of the RNAs. The mP-SF and mE-SF RNAs analyzed in Figure 1 were designed to favor formation of the correct structure for editing (Fig. 1); in contrast, the replicating fl-E and fl-P RNAs examined in Figure 2 must adopt at least two secondary structures: the branched structure required for editing and the unbranched rod structure required for RNA replication. Previous analysis of the secondary structures of mE RNA using computational methods and in vitro approaches indicated that the branched structure is less stable energetically than the unbranched rod, yet a substantial fraction of the RNA folds into this metastable structure following transcription in vitro (Linnstaedt et al. 2006). Thus, the extent to which the RNA forms the branched structure required for editing following transcription could be an important determinant of how much editing occurs. We explored this possibility by comparing the folding properties of $\mathrm{mE}$ and $\mathrm{mP}$ RNAs using the RNA massively parallel genetic folding algorithm, MPGAfold.

MPGAfold analysis was performed using two sets of conditions: full-length sequence folds, in which secondary structures formed by the entire sequence evolve during the course of a run; and "co-transcriptional" folds, in which the sequence available for folding is extended by $5 \mathrm{nt}$ per generation. Based on 100 runs per population size, with population sizes ranging from $4 \mathrm{~K}$ to $64 \mathrm{~K}$, these analyses predicted several types of secondary structures for mP RNA (Fig. 3). These structures include the most energetically stable unbranched rod (Fig. 3, A1), as well as several branched conformations (Fig. 3, $\mathrm{B}_{\mathrm{EDIT}}, \mathrm{B}_{\mathrm{ALT1}}, \mathrm{B}_{\mathrm{ALT2}}$, $\left.\mathrm{B}_{\mathrm{ALT3}}\right)$. The $\mathrm{B}_{\mathrm{EDIT}}$ structure is nearly identical to that proposed for editing $\mathrm{mE}$ RNA (Linnstaedt et al. 2006), and the comparable $\mathrm{mE}$ structure is actually in better agreement with the RNase digestion secondary structure mapping data obtained for $\mathrm{mE}$ (Linnstaedt et al. 2006). Representative statistics for 16K population MPGAfold run solutions are shown in Table 1, with structures categorized as $\mathrm{B}_{\mathrm{EDIT}}$, other branched structures (e.g., $\mathrm{B}_{\mathrm{ALT} 1}, \mathrm{~B}_{\mathrm{ALT} 2}$, $\mathrm{B}_{\mathrm{ALT3}}$ ), and unbranched structures (A1 and similar structures that include short cruciform stems).

Both the full-length and co-transcriptional MPGAfold predictions showed striking differences in the folding tendencies of the $\mathrm{mP}$ and $\mathrm{mE}$ RNAs. Overall, $\mathrm{mP}$ was markedly less likely to assume branched conformations than $\mathrm{mE}$ (Table 1). In full-length runs, $\mathrm{mP}$ converged more rapidly to the final unbranched rod configuration, and exhibited a shorter-lived $\mathrm{B}_{\text {EDIT }}$ conformation than $\mathrm{mE}$ (Fig. 4). Only the lowest run population size tested (4K) produced branched structures of different types and only in 


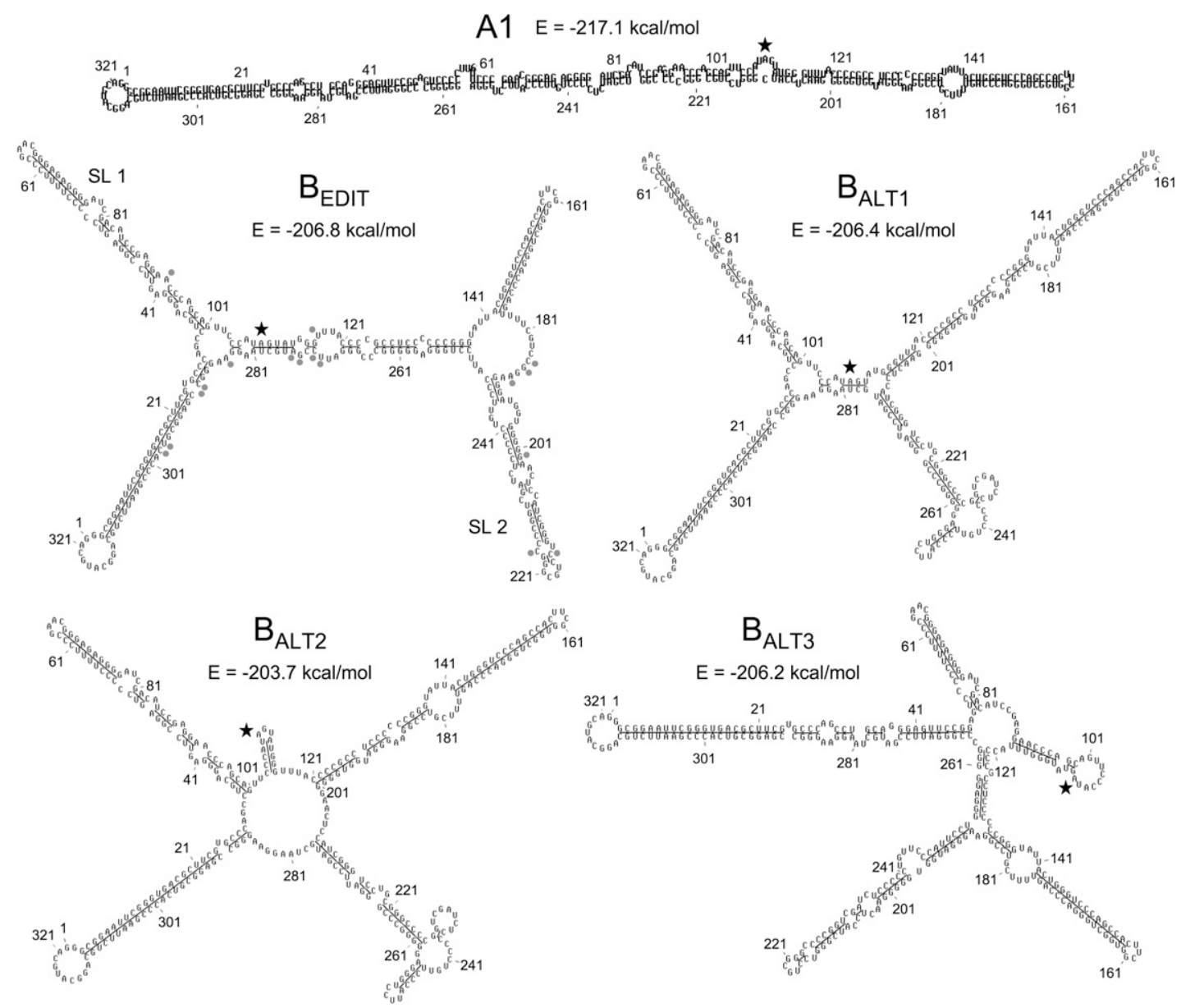

FIGURE 3. Secondary structures of the HDV-3 mP RNA predicted by MPGAfold. Black stars denote the location of the amber/W editing site in each predicted structure. The unbranched rod A1 is the best fit structure (lowest free energy structure achieved in MPGAfold runs). Structure $\mathrm{B}_{\mathrm{EDIT}}$ is very similar to that previously predicted for the Ecuadorian RNA mE (Linnstaedt et al. 2006) using efn2 energy rules. Extensions, or overruns, of the terminal stems in alternative branched structures $\mathrm{B}_{\mathrm{ALT} 1}, \mathrm{~B}_{\mathrm{ALT} 2}$, and $\mathrm{B}_{\mathrm{ALT} 3}$ alter base-pairing around the amber/W site and the lengths of stem-loops SL1 and SL2. Gray dots shown in the BEDIT structure denote locations of sequence differences between the $\mathrm{mP}$ and $\mathrm{mE}$ RNAs. Free energies predicted by MPGAfold are indicated. Energy calculations used the efn energy rules only, without the coaxial stem-stacking energy calculations for multibranch loops (efn2) (Mathews et al. 1999).

$7 \%$ of final solutions for $\mathrm{mP}$. No branched structures were observed as final solutions for $\mathrm{mP}$ in $16 \mathrm{~K}$ population runs (Table 1), which converged to rod-like structures such as A1. In a representative $16 \mathrm{~K}$ population run (Fig. 4B), unbranched rod and rod-like structures dominated, with the $\mathrm{B}_{\mathrm{EDIT}}$ conformer appearing only briefly. In contrast to the behavior of the $\mathrm{mP}$ sequence, $\mathrm{mE} \mathrm{B}_{\mathrm{EDIT}}$ conformers dominated the distribution of predicted secondary structures in $\mathrm{mE}$ runs with population sizes of $16 \mathrm{~K}$ or lower (Table 1). This dominance yielded to unbranched structures (e.g., A1) in higher population runs, but persisted at a level of $14 \%$ even at $64 \mathrm{~K}$, the highest population run size tested. Even in runs that yielded unbranched rod structures as final solutions, such as the $16 \mathrm{~K}$ population run shown in Figure $4 \mathrm{~A}$, the $\mathrm{B}_{\mathrm{EDIT}}$ conformer persisted over many generations before yielding to the rod-like structure.

TABLE 1. Percent of full-length and co-transcriptional 16K MPGAfold runs yielding indicated types of secondary structures for $\mathrm{mP}$ and $\mathrm{mE}$ RNAs

\begin{tabular}{llccc}
\hline & & \multicolumn{3}{c}{ Type of secondary structure } \\
\cline { 3 - 5 } Sequence & Type of MPGAfold run & B EDIT & Alternative branched $^{\text {a }}$ & Unbranched rod $^{\text {b }}$ \\
\hline $\mathrm{mP}$ & Full length & 0 & 0 & 100 \\
$\mathrm{mE}$ & Full length & 56 & 3 & 41 \\
$\mathrm{mP}$ & Co-transcriptional & 19 & 74 & 7 \\
$\mathrm{mE}$ & Co-transcriptional & 73 & 27 & 0 \\
\hline
\end{tabular}

${ }^{\mathrm{a}}$ For example, $\mathrm{B}_{\mathrm{ALT1}}, \mathrm{B}_{\mathrm{ALT} 2}$, and $\mathrm{B}_{\mathrm{ALT} 3}$ (Fig. 3).

Includes structure A1 as well as mostly unbranched rod structures similar to those previously reported (Linnstaedt et al. 2006). 

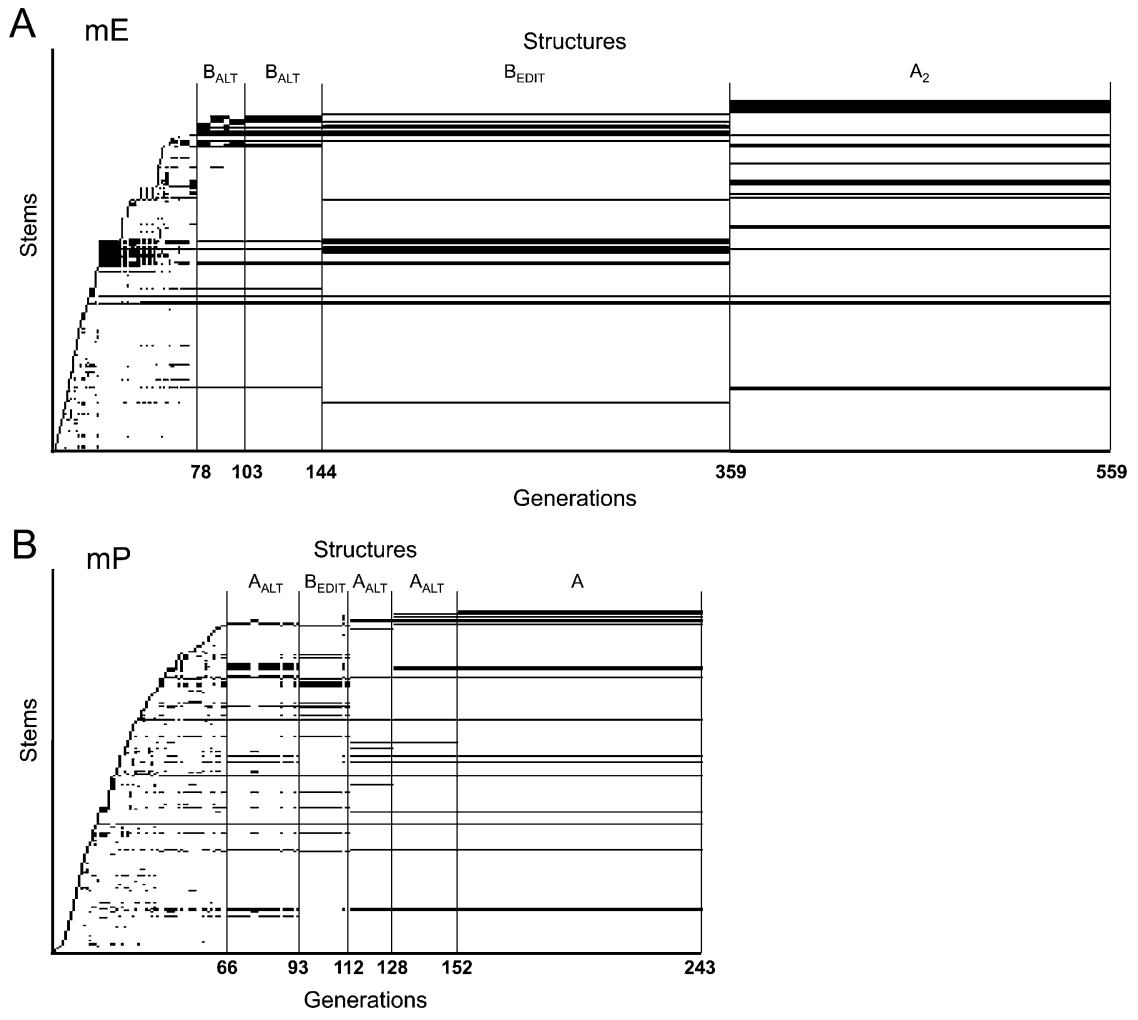

FIGURE 4. Stem Trace plots illustrating maturation of $\mathrm{mE}$ and $\mathrm{mP}$ structures in $16 \mathrm{~K}$ population MPGAfold runs. The population consensus structures are depicted at each run generation, indicated numerically along the horizontal axis, and mature from left to right. Individual secondary structure stem elements (defined by $5^{\prime}$ start position, $3^{\prime}$ stop position, and number of base pairs) are arranged along the vertical axis, in order of their appearance in the consecutive predicted consensus structures. Longer horizontal bars indicate the presence of a stem over more generations of a run. The full secondary structure for a generation corresponds to the set of stems with the same $x$ coordinate. Thin vertical lines demarcate stages of maturation during which run generations were dominated by particular structures that are identified by labels above the plot. (A) $16 \mathrm{~K}$ population folding run for mE RNA. The final state, labeled A2, is a rod structure identical to that previously published (Linnstaedt et al. 2006, Fig. 2 therein). (B) 16K population folding run for mP RNA. Horizontal scales have been adjusted for clarity. Structures marked as $\mathrm{A}_{\mathrm{ALT}}$ are similar to mostly unbranched rod structures previously reported (Linnstaedt et al. 2006), in that they contain a few short side branches. The final structure of this run, labeled $\mathrm{A}$, is also a near-rod conformer similar to one previously observed for $\mathrm{mE}$ (Linnstaedt et al. 2006, Fig. 2 therein, structure A1). As a result of the different free energy landscapes of $\mathrm{mE}$ and $\mathrm{mP}$, the $16 \mathrm{~K}$ full-length runs for $\mathrm{mP}$ RNA exhibit a shorter-lived transitional $\mathrm{B}_{\mathrm{EDIT}}$ conformation and converge more rapidly to the final state, as compared with runs for $\mathrm{mE}$.

Co-transcriptional folding helps to identify intermediate structures by delaying the global optimization of the folding and may mimic the ability of the RNA to fold as it is being transcribed. With this method, branched structures dominated solutions for both $\mathrm{mE}$ and $\mathrm{mP}$ sequences (Table $1)$; this dominance was observed even for the largest population runs. Nevertheless, the results for $\mathrm{mP}$ and $\mathrm{mE}$ differed substantially in the types of branched conformations formed (Table 1). For $\mathrm{mE}$, the overwhelming majority of runs produced a branched structure very similar to that previously identified (Linnstaedt et al. 2006). For example, $16 \mathrm{~K}$ runs produced about a 3:1 ratio of branched editing structures to alternative branched structures (Table 1). In contrast, the most common structures for $\mathrm{mP}$ were alternative branched structures (Table 1) of the types shown in Figure $3\left(\mathrm{~B}_{\mathrm{ALT1}}, \mathrm{B}_{\mathrm{ALT} 2}, \mathrm{~B}_{\mathrm{ALT} 3}\right)$. These alternative branched structures are not likely to serve as efficient substrates for amber/W site editing because either the predicted base-pairing of the editing site itself is dramatically altered (Fig. 3, $\left.\mathrm{B}_{\mathrm{ALT} 2}, \mathrm{~B}_{\mathrm{ALT} 3}\right)$ or substantial branching occurred as close as 4 nt away from the 3 ' side of the site (Fig. 3, $\mathrm{B}_{\mathrm{ALT1}}$ ). Overall, the MPGAfold results indicate that $\mathrm{mP}$ RNA differs significantly from $\mathrm{mE}$ in its ability to form the $\mathrm{B}_{\mathrm{EDIT}}$ structure required for editing.

The MPGAfold analysis indicated that differences in the free energy folding landscapes of $\mathrm{mP}$ RNA and $\mathrm{mE}$ RNA affect their relative abilities to form the branched structure required for editing in two ways. First, the unbranched rod structure (Fig. 3, A1) is favored substantially more for $\mathrm{mP}$ RNA compared with $\mathrm{mE}$ (Table 1). This difference is attributed to the deeper free energy well of $\mathrm{mP}$ compared with $\mathrm{mE}$ (predicted free energies of -217.1 $\mathrm{kcal} / \mathrm{mol}$ and $-195.4 \mathrm{kcal} / \mathrm{mol}$, respectively, for the unbranched rod structure A1) and to the greater difference between the predicted free energies of the $\mathrm{A} 1$ and $\mathrm{B}_{\mathrm{EDIT}}$ structures for $\mathrm{mP}$ than for $\mathrm{mE}(10.3 \mathrm{kcal} / \mathrm{mol}[4.7 \%]$ for $\mathrm{mP}$ versus $4.2 \mathrm{kcal} / \mathrm{mol}$ [2.1\%] for $\mathrm{mE}$ ). Second, among potential branched structures, $\mathrm{B}_{\mathrm{EDIT}}$ is favored in predictions of $\mathrm{mE}$, but alternative branched structures dominate predictions for $\mathrm{mP}$ RNA. Examination of individual cotranscriptional folds (results not illustrated) and full domain folds, illustrated in Figure 4B, revealed that the $\mathrm{mP} \mathrm{B}_{\mathrm{EDIT}}$ structure is often one of several alternative branched and less fit near-rod conformers with the same free energy (results not illustrated), which indicates the existence of multiple folding states directly competing in the overall $\mathrm{mP}$ energy landscape.

\section{mP RNA forms the branched editing structure less efficiently than the mE RNA following transcription in vitro}

Previously, we found that mE RNA migrates as two distinct bands on a nondenaturing polyacrylamide gel following 
transcription in vitro with T7 RNA polymerase (Linnstaedt et al. 2006). RNA in the more slowly migrating band formed the metastable branched structure that is the substrate for editing; the more rapidly migrating band contained RNA that had assumed the most energetically stable structure- the unbranched rod. In order to analyze whether the different folding dynamics of $\mathrm{mP}$ and $\mathrm{mE}$ RNAs predicted by MPGAfold translated into different abilities of these RNAs to form the branched editing structure in vitro, we analyzed the structural distributions of $\mathrm{mP}$ and $\mathrm{mE}$ RNAs using nondenaturing gel electrophoresis following transcription with $\mathrm{T} 7$ polymerase. Because the rate of transcription can affect RNA folding dynamics (Repsilber et al. 1999; Diegelman-Parente and Bevilacqua 2002), we performed transcription reactions at $20^{\circ} \mathrm{C}$ with $100 \mu \mathrm{M}$ NTPs. Under these conditions the rate of transcription by T7 polymerase is reduced considerably (Repsilber et al. 1999) and is likely to be more similar to the rate that HDV RNA is transcribed in infected cells.

Electrophoresis of in vitro-transcribed mP RNA yielded two major bands with migrations similar to those of the branched and unbranched conformations of mE RNA (Fig. $5 \mathrm{~A}$, left). Similar to the results obtained for RNAs that were mutated to favor the branched conformation, the branched $\mathrm{mP}$ RNA was edited more efficiently than branched $\mathrm{mE}$ RNA (Fig. 5B). Consistent with the MPGAfold analysis, compared with $\mathrm{mE}, \mathrm{mP}$ RNA preferentially formed unbranched rather than branched structures. The correlation with the MPGAfold results was further improved by extension of the electrophoresis time, which resolved branched $\mathrm{mP}$ RNA as two bands, S1 and S2 (Fig. 5A, right). $\mathrm{S} 1$ co-migrated with the branched species formed by mE RNA, for which no clear additional bands were observed (Fig. 5A); S1 also co-migrated with mP-SF RNA (data not shown). In order to determine whether the two branched species formed by $\mathrm{mP}$ RNA could be edited, RNAs in bands S1 and S2 were isolated from the polyacrylamide gel and assayed for editing by ADAR1. RNA in the S1 band was efficiently edited and no editing was detected for RNA in the S2 band (Fig. 5C). We conclude that RNA in the mP S1 band is most likely in the $\mathrm{B}_{\mathrm{EDIT}}$ structure (Fig. 3) because (1) it is efficiently edited; (2) S1 migrates with the same mobility as the branched structure formed by mE RNA, which has been shown to be $B_{\text {EDIT }}$ (Linnstaedt et al. 2006); (3) mP S1 RNA migrates identically with mP-SF RNA; (4) another mP RNA construct (data not shown), in which the stems near the bases of SL1 and SL2 were further stabilized by an additional set of stem-flip mutations, also migrates identically with S1 and is edited with the same efficiency as mP-SF. The difference in editing between S1 and mP-SF (Fig. 1) is most likely due to the metastable nature of $\mathrm{mP}$ in the branched structure isolated from S1; analysis of the mobility of the RNA after incubation with ADAR1 indicated that about half of the RNA in S1 had changed to the unbranched rod conforma- tion, which is not edited. RNA in the S2 band, which is not a substrate for efficient editing (Fig. 5C), most likely includes RNAs in other branched conformations, such as those predicted by MPGAfold (Fig. 3, $\mathrm{B}_{\mathrm{ALT1} 1}-\mathrm{B}_{\mathrm{ALT} 3}$ ).

The results of the analysis of secondary structures formed following transcription in vitro (Fig. 5) show remarkably good qualitative agreement with the RNA folding dynamics predicted by MPGAfold. The algorithm indicated that, compared with $\mathrm{mE}, \mathrm{mP}$ RNA favors the formation of the unbranched rod structure rather than branched conformations (Table 1). Following transcription in vitro, although mP RNA was about evenly divided between branched and unbranched structures, it formed branched structures less efficiently than $\mathrm{mE}$ (Fig. 5). Furthermore, MPGAfold indicated that $\mathrm{mP}$ tended to form alternative branched conformations rather than $\mathrm{B}_{\mathrm{EDIT}}$ (Fig. 3; Table 1), whereas mE RNA preferentially formed this structure. This prediction was also borne out in vitro. Following transcription in vitro, $\mathrm{mP}$ RNA formed both the branched editing structure $\mathrm{B}_{\mathrm{EDIT}}$ as well as alternative branched structures that could not be edited; on the other hand, mE RNA

A
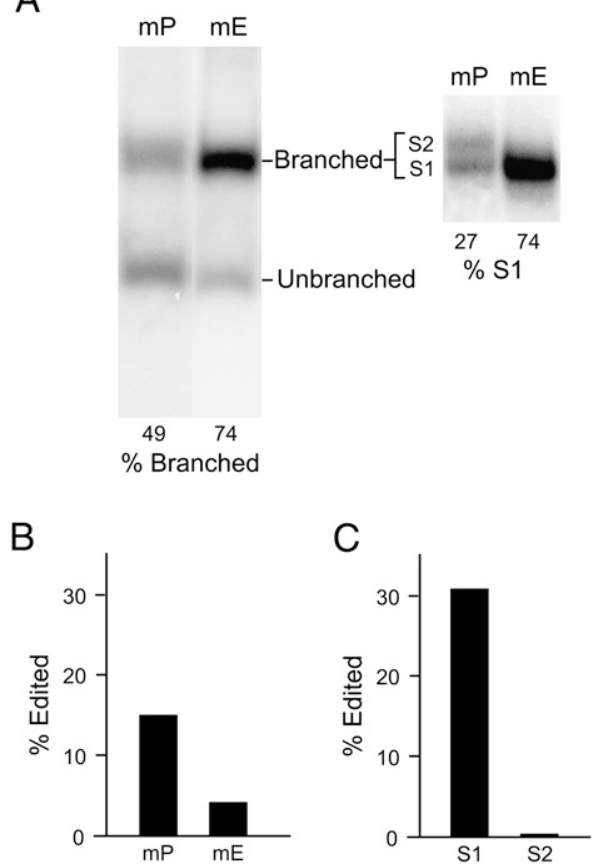

FIGURE 5. Comparison of the secondary structure distribution of Ecuadorian and Peruvian RNAs following transcription in vitro. $(A)$ $\mathrm{mP}$ and $\mathrm{mE}$ RNAs were transcribed in vitro using T7 RNA polymerase and electrophoresed on a $6 \%$ nondenaturing polyacrylamide gel for 4 $\mathrm{h}$ at $4^{\circ} \mathrm{C}$ (left). The locations of branched and unbranched RNAs are indicated. Electrophoresis for $8 \mathrm{~h}$ at $4^{\circ} \mathrm{C}$ resolved two branched structures, S1 and S2, for mP RNA, but not for $\mathrm{mE}$ (Right). The migration of $\mathrm{S} 1$ was the same as the single branched species formed by mE RNA. (B) Editing of branched $\mathrm{mP}$ and $\mathrm{mE}$ RNAs. Branched $\mathrm{mP}$ and $\mathrm{mE}$ RNAs were gel-isolated and assayed for amber/W site editing as in Figure 1. (C) S1 and S2 mP RNAs were isolated from the gel in $A$ and assayed for amber/W site editing as in Figure 1. 
formed only $\mathrm{B}_{\mathrm{EDIT}}$ (Fig. 5). Overall, only $27 \%$ of mP RNA forms the $\mathrm{B}_{\mathrm{EDIT}}$ structure, whereas $74 \%$ of $\mathrm{mE}$ RNA forms $\mathrm{B}_{\text {EDIT }}$ - $\mathrm{a}$ threefold difference (Fig. 5A, right). Other transcription conditions (higher temperature, higher NTP concentrations) that could have affected the rate of RNA synthesis by $\mathrm{T} 7$ polymerase yielded similar results in that the efficiency of $\mathrm{B}_{\mathrm{EDIT}}$ formation was always greater for $\mathrm{mE}$ than for $\mathrm{mP}$ (data not shown).

These results suggest an explanation for the observations that, in vitro, mP RNA, derived from the Peruvian isolate, was edited more efficiently than the Ecuadorian isolatederived mE RNA but, in cells, replicating Peruvian RNA was edited less efficiently than replicating Ecuadorian RNA (Fig. 6). Although the branched structure formed by Peruvian RNA is a better substrate for editing than Ecuadorian RNA (Fig. 1), the Peruvian RNA forms less of this structure following transcription (Fig. 5; Table 1). The net result of these two counteracting effects is that, overall, editing levels are lower for the Peruvian isolate (Fig. 2).

\section{DISCUSSION}

Our comparison of amber/W site editing in two HDV-3 isolates, one from Peru and one from Ecuador, has indicated that at least two factors combine to determine the level of editing in this genotype. One determinant is the activity of the editing site in the $\mathrm{B}_{\text {EDIT }}$ structure. Primarily due to four sequence variations in the base-paired region 4 nt 3 ' from the amber/W editing site, the in vitro editing activities of the Ecuadorian and Peruvian $B_{\text {EDIT }}$ structures differ by three- to fourfold (Fig. 1). This variation in editing would be substantial were it to carry over to replication in cells, because we have previously shown that mutations that alter editing levels by about fourfold can have dramatic

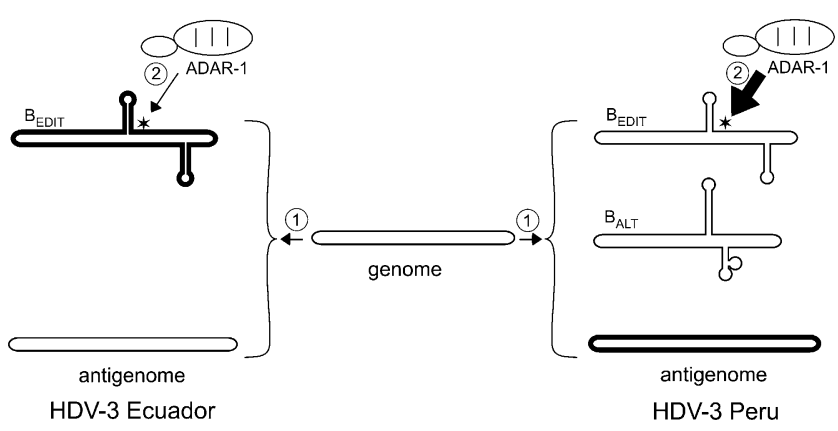

FIGURE 6. Model depicting factors that control amber/W site editing in HDV-3. The unbranched rod structure is indicated by an elongated rounded rectangle; branched structures are labeled $\mathrm{B}_{\mathrm{EDIT}}$ and $\mathrm{B}_{\mathrm{ALT}}$. The first control point occurs following the synthesis of the antigenome, which folds into different structures. Ecuadorian HDV-3 preferentially forms the $\mathrm{B}_{\mathrm{EDIT}}$ structure. In comparison, HDV-3 Peru forms the unbranched rod, as well as additional branched structure(s) $\left(\mathrm{B}_{\mathrm{ALT}}\right)$ not likely to be edited. The second control point is the activity of ADAR1 on the amber/W site in the $\mathrm{B}_{\text {EDIT }}$ structure. $\mathrm{B}_{\mathrm{EDIT}}$ of HDV3 Peru is edited more efficiently than $\mathrm{B}_{\mathrm{EDIT}}$ of HDV-3 Ecuador. effects on RNA replication and virus production (Jayan and Casey 2005). However, the different editing activities of the Peruvian and Ecuadorian RNAs are countered by a second determinant of editing levels - the variable abilities of these RNAs to fold into the metastable $B_{\text {EDIT }}$ structure following transcription. In silico analysis of RNA folding with the MPGAfold algorithm indicated that the Peruvian RNA is much less able to form the branched structure required for editing than is the Ecuadorian RNA (Fig. 4; Table 1). This prediction is supported by analysis of RNA folding following transcription in vitro. As a result of the combined effects of the variable $B_{\text {EDIT }}$ activities and the RNA structural dynamics, the replicating Ecuadorian RNA is edited more efficiently.

Certainly, the folding of HDV RNA during HDV replication in cells may be affected by numerous factors not present in our in vitro system, and it may be interesting in future experiments to examine the contributions of HDAg and host RNA chaperones to the folding process for HDV RNA. In this light, the agreement between the folding patterns predicted by the MPGAfold analysis and the in vitro folding of the $\mathrm{mP}$ and $\mathrm{mE}$ RNAs following transcription is remarkable. MPGAfold not only identified the branched editing structures for both HDV-3 isolates as likely metastable states, but also predicted the differences in the observed fraction of the RNAs that formed these structures in vitro.

The particular folding pathways used by $\mathrm{mP}$ and $\mathrm{mE}$ RNAs to achieve the functional branched editing and unbranched rod structures (Fig. 3, $\mathrm{B}_{\mathrm{EDIT}}$, A1, respectively) are not yet known, but the MPGAfold analysis suggests that several sequence variations affect their different folding behaviors and which conformations are favored. Sequence variations in $\mathrm{mP}$, relative to $\mathrm{mE}$, decreased the stability of the base (proximal) stems of SL1 and SL2 and increased the stability of the base-paired stems that extend from the two opposite ends of the structure. Thus, the stem that ends in the $5^{\prime}$ and $3^{\prime}$ ends of the alternative branched structure $\mathrm{B}_{\mathrm{ALT3}}$ (Fig. 3) extends at the expense of SL1, and the stem that ends in the loop beginning at position 158 (Fig. 3, $\mathrm{B}_{\mathrm{ALT} 1}, \mathrm{~B}_{\mathrm{ALT} 2}$ ) extends at the expense of SL2. These stem extensions, or overruns, drive the transformation of the branched structures into the final, most stable unbranched rod structures (Fig. 3, A1). Thus, it seems probable that the observed higher propensity for the folding "overrun" of the motifs described above is responsible for the significant domination of the alternative branched and linear conformations over the branched editing structures in $\mathrm{mP}$. Nevertheless, analysis of the effects of sequence substitutions at several locations (data not shown) indicated that the different folding behaviors of $\mathrm{mE}$ and $\mathrm{mP}$ RNAs could not be definitively localized to just a few nucleotide differences or clusters of differences. Thus, the folding dynamics are likely to be complex and involve several elements of the structure. 
In our previous MPGAfold analysis of mE RNA, which identified the metastable branched structure required for editing, as well as the unbranched rod structure, we employed the coaxial stem-stacking energy calculations for multibranch loops (efn2) (Mathews et al. 1999) at every generation within runs of the algorithm. In the current study, we obtained results more consistent with in vitro and in vivo studies without applying these energy rules, particularly for $\mathrm{mP}$. With the efn2 calculations included, we were unable to detect the branched editing structure for $\mathrm{mP}$, even though previous mutational and structural analyses indicated that a branched structure such as $B_{\mathrm{EDIT}}$ is required for editing (Casey 2002; Linnstaedt et al. 2006). The $B_{\text {EDIT }}$ structure was readily observed when the efn2 calculations were left out (Figs. 3, 4; Table 1). Moreover, for both $\mathrm{mE}$ and $\mathrm{mP}$, the $\mathrm{B}_{\mathrm{EDIT}}$ structure identified in this way was in better agreement with the enzymatic structural analysis (Linnstaedt et al. 2006), further confirming the validity of our approach. The most important effect of omitting the efn2 calculations was a flatter energy landscape-the predicted free energies of rodlike structures were minimally affected, while those of branched structures such as $\mathrm{B}_{\text {EDIT }}$ became more negative. Because the evolution of structures in the algorithm is affected by energy differences between states, the flatter energy landscape results in more gradual transitions from branched to cruciform (intermediate) to rod-like conformations. The differences between the $\mathrm{mP}$ and $\mathrm{mE}$ energy landscapes, however, remained visible, which is illustrated in the different convergence rates and transitional states captured in Figure 4.

The analysis of the structural dynamics of the Ecuadorian and Peruvian HDV-3 RNAs suggests that the folding of the antigenome is stochastic to a degree that is incorporated into its function. Only a fraction of HDV antigenome RNA forms the branched editing structure $\mathrm{B}_{\mathrm{EDIT}}$ following transcription. Because only this fraction can be edited, it is a critical factor in establishing the level of editing that occurs. This fractional folding may differ from the behavior of other RNAs that are capable of forming multiple structures with distinct functions (e.g., Tucker and Breaker 2005; Chadalavada et al. 2007) in that, in most instances, particular structures are formed under a given set of conditions in order to perform a particular function. For example, the HDV ribozyme, which efficiently folds into the structure required for cleavage following transcription (Diegelman-Parente and Bevilacqua 2002; Chadalavada et al. 2007), must form the unbranched rod structure after cleavage and ligation so that subsequent transcription can occur.

Our observations expand the possibilities for functionally important roles for RNA folding dynamics. It seems likely that fractional folding will be important for other structured RNAs, particularly for viral RNAs that have multiple functions and for RNAs that are edited or alternatively spliced; in such cases the fraction of RNA that exhibits one particular functional activity versus another may be particularly important.

Finally, we note that, while editing by ADAR is highly specific for adenosines in particular structural contexts, the specific structural features required for editing have not been fully determined. Nor is it understood how sequence and structural variations among editing sites contribute to differences in editing activity. Given the differences in the editing activities of the Ecuadorian and Peruvian $\mathrm{B}_{\mathrm{EDIT}}$ structures that we report here, further comparative studies of the RNA structures in the vicinity of the amber/W editing sites of these two HDV isolates are likely to advance our understanding of the RNA structural requirements for editing.

\section{MATERIALS AND METHODS}

\section{Plasmid construction}

Constructs pMD-III-2 and pMD-III-2SF, used for in vitro synthesis of miniaturized RNAs derived from an Ecuadorian HDV isolate, have been described previously (Linnstaedt et al. 2006); here, we refer to the RNAs transcribed from these constructs as $\mathrm{mE}$ and $\mathrm{mE}-\mathrm{SF}$, respectively. $\mathrm{mE}-\mathrm{SF}$ was designed with stem-flip mutations that favor the formation of the branched secondary structure (Linnstaedt et al. 2006). We created constructs for the synthesis of miniaturized RNAs derived from the Peruvian HDV-3 isolate (Casey et al. 1993) using the same methods; we refer to these RNAs as $\mathrm{mP}$ and $\mathrm{mP}-\mathrm{SF}$. The sequence of the $\mathrm{mP}$ RNA can be accessed using GenBank accession number FJ743689. Sitedirected mutagenesis was used to create mutant constructs in which sequence differences at three locations (edit, proximal, and distal) were exchanged between the Ecuadorian and Peruvian miniaturized RNAs. The locations exchanged were: edit, positions 272, 273, 275, and 276; proximal, positions 202, 216, 224; distal, positions 185, 187, 188 (using the numbering of mP RNA). RNAs were named based on the parent sequence plus the source of the exchanged nucleotides; thus, $\mathrm{mE}-\mathrm{SF} \rightarrow \mathrm{P}_{\mathrm{EDIT}}$ contains miniaturized Ecuadorian sequences in which the four sequence variations from the Peruvian RNA near the editing site have been substituted for Ecuadorian sequences. Construct pHDV-III-NR M24/25 expresses a nonreplicating HDV-3 Peru RNA in which the secondary structure of the edited conformation around the amber/W site is energetically favored by having removed SL1 and SL2 sequences (Cheng et al. 2003); we refer to that expression construct here as Pnr. In the construct Pnr $\rightarrow \mathrm{P}_{\mathrm{EDIT}}$, the $4 \mathrm{nt}$ in Pnr corresponding to positions 272, 273, 275, and $276 \mathrm{in} \mathrm{mP}$ (Fig. 1) were changed to the Ecuadorian sequence.

The construct $\mathrm{pHDV} \bullet \mathrm{III}(+)$ produces a 1.2 -mer HDV RNA that initiates replication in transfected Huh-7 cells (Casey and Gerin 1998); the sequences in this construct are derived from the Peruvian isolate, which is the HDV-3 prototype. Here, we refer to this full-length expression construct as fl-P. In order to compare editing between replicating Peruvian and Ecuadorian RNAs, we used PCR amplification and standard cloning methods to create a replicating construct, fl-E, in which sequences from the Ecuadorian isolate replaced those of the Peruvian isolate at positions 9771097 and 499-616 in fl-P; these regions together make up the editing site. 


\section{In vitro transcription}

mE-SF, mP-SF RNAs, and derivatives were transcribed in vitro from linearized templates using T7 polymerase (New England Biolabs), as described (Linnstaedt et al. 2006). Transcription conditions for $\mathrm{mE}, \mathrm{mP}$ RNAs and derivatives were chosen to reduce the transcription rate of T7 polymerase, which is considerably faster than pol II, to $\sim 12 \mathrm{nt} / \mathrm{sec}$ (Repsilber et al. 1999). Briefly, linearized plasmids were transcribed for $1 \mathrm{~h}$ at $25^{\circ} \mathrm{C}$ in a $20 \mu \mathrm{L}$ reaction mixture containing $40 \mathrm{mM}$ Tris- $\mathrm{HCl}(\mathrm{pH}$ 7.9), 6 $\mathrm{mM} \mathrm{MgCl} 2,10 \mathrm{mM}$ dithiothreitol, $2 \mathrm{mM}$ spermidine, $40 \mathrm{U}$ T7 RNA polymerase, and $120 \mu \mathrm{M}$ of each nucleoside triphosphate (ATP, CTP, GTP, UTP). RNA was electrophoresed at $4^{\circ} \mathrm{C}$ on a $6 \%$ polyacrylamide gel precooled to $4^{\circ} \mathrm{C}$.

\section{Editing analysis}

For in vitro editing reactions, one femtomole of gel-purified, in vitro transcribed RNA ( $\mathrm{mP}, \mathrm{mE}, \mathrm{mP}-\mathrm{SF}$, or $\mathrm{mE}-\mathrm{SF}$ ) was incubated with nuclear extract from HEK293T cells transfected with an ADAR1 expression construct, as described (Linnstaedt et al. 2006). RNA editing at the amber/W site was detected by Sty I digestion of RT-PCR products as previously described (Casey and Gerin 1995; Polson et al. 1996; Jayan and Casey 2002b; Linnstaedt et al. 2006). Amber/W site editing in HDV RNA isolated from transfected Huh-7 cells was determined as before (Cheng et al. 2003). For analysis of amber/W site editing in viral RNA in patient sera, RNA was isolated by proteinase $\mathrm{K}$ digestion, extraction, and ethanol precipitation, as described (Niro et al. 1997); editing at the amber/ W site was determined by RT-PCR amplification using primers 5'-GAAGGAAGGCCCTCGAGAACAAGA-3' and 5'-GAGATGC CATGCCGACCCGAAGAG-3' followed by ${ }^{32} \mathrm{P}$ labeling and Sty I digestion, as described (Polson et al. 1996).

\section{Analysis of RNA in transfected cells}

Huh-7 cells were cultured in Dulbecco's modified Eagle's medium supplemented with $10 \%$ fetal bovine serum and $1 \mathrm{mM}$ glutamine. Cells were plated in 12-well dishes and transfected with $1 \mu \mathrm{g}$ of the indicated plasmid DNAs using Lipofectamine Plus reagent (Invitrogen) according to the manufacturer's specifications. Total cellular RNA was harvested at indicated times between 3 and 12 d post-transfection using Qiashredder and the RNeasy kit (Qiagen) according to the manufacturer's recommended procedures. RNA editing was analyzed by Sty I digestion of reverse transcription-PCR (RT-PCR) products as described previously (Casey and Gerin 1995; Polson et al. 1996; Casey 2002). For quantifying RNA levels by blot hybridization, RNA was electrophoresed through $1.5 \%$ agarose gels containing $2.2 \mathrm{M}$ formaldehyde, transferred to positively charged nylon membranes, and hybridized with an antigenomic-sense ${ }^{32} \mathrm{P}$-labeled probe, as described previously (Jayan and Casey 2005). Relative levels of HDV RNA were determined by radioanalytic scanning of blots with a Molecular Dynamics Storm PhosphoImager. All experiments were repeated at least once and included duplicate transfections.

\section{Computational analysis of RNA secondary structure comparisons}

The prediction of the potential secondary structures of mP RNA was performed by our massively parallel genetic algorithm,
MPGAfold (Shapiro and Navetta 1994; Shapiro and Wu 1996; Shapiro et al. 2001a,b). We have demonstrated in previous studies that this stochastic algorithm focuses on a few most significant RNA conformations, and that it can also identify biologically significant folding intermediates (Shapiro et al. 2001a; Kasprzak et al. 2005; Gee et al. 2006). In short, given the primary nucleotide sequence, the algorithm evolves a population of RNA secondary structures using their free energy as the fitness criterion. This is performed in parallel, with the size of the maturing population set by the user. Since the algorithm is stochastic, multiple runs are performed to obtain the overall consensus results. The population size is an important parameter. Smaller population runs (i.e., $4 \mathrm{~K}$ or $8 \mathrm{~K}$ ) more frequently converge to lower fitness (i.e., higher free energy) conformers, which may appear as intermediate or metastable conformations in higher population runs ( $64 \mathrm{~K}$ for the sequences considered here, or higher for longer input sequences). In this study, the various branched conformers (Fig. 3) are such intermediates. Another feature employed in this study is cotranscriptional folding, i.e., folding of an elongating sequence. Since in this mode the folding structures evolve in a changing context, transitional motifs are more likely to form and persist longer. As a result alternative folding pathways can be explored. The analysis of MPGAfold results was performed with the help of our software workbench StructureLab (Shapiro and Kasprzak 1996; Kasprzak and Shapiro 1999; Shapiro et al. 2006).

\section{ACKNOWLEDGMENTS}

This work was supported by grant R01-AI42324 from the National Institute of Allergy and Infectious Diseases, National Institutes of Health, and with Federal funds from the National Cancer Institute, National Institutes of Health, under Contract No. NO1-CO-12400. This research was supported, in part, by the Intramural Research Program of the NIH, National Cancer Institute, Center for Cancer Research. The content of this publication does not necessarily reflect the views or policies of the Department of Health and Human Services, nor does mention of trade names, commercial products, or organizations imply endorsement by the U.S. Government.

Received December 9, 2008; accepted January 20, 2009.

\section{REFERENCES}

Aruscavage, P.J. and Bass, B.L. 2000. A phylogenetic analysis reveals an unusual sequence conservation within introns involved in RNA editing. RNA 6: 257-269.

Bass, B.L. 2002. RNA editing by adenosine deaminases that act on RNA. Annu. Rev. Biochem. 71: 817-846.

Bhalla, T., Rosenthal, J.J., Holmgren, M., and Reenan, R. 2004. Control of human potassium channel inactivation by editing of a small mRNA hairpin. Nat. Struct. Mol. Biol. 11: 950-956.

Casey, J.L. 2002. RNA editing in hepatitis delta virus genotype III requires a branched double-hairpin RNA structure. J. Virol. 76: 7385-7397.

Casey, J.L. 2006. RNA editing in hepatitis delta virus. Curr. Top. Microbiol. Immunol. 307: 67-89.

Casey, J.L. and Gerin, J.L. 1995. Hepatitis D virus RNA editing: Specific modification of adenosine in the antigenomic RNA. J. Virol. 69: 7593-7600. 
Casey, J.L. and Gerin, J.L. 1998. Genotype-specific complementation of hepatitis delta virus RNA replication by hepatitis delta antigen. J. Virol. 72: 2806-2814.

Casey, J.L., Bergmann, K.F., Brown, T.L., and Gerin, J.L. 1992. Structural requirements for RNA editing in hepatitis delta virus: Evidence for a uridine-to-cytidine editing mechanism. Proc. Natl. Acad. Sci. 89: 7149-7153.

Casey, J.L., Brown, T.L., Colan, E.J., Wignall, F.S., and Gerin, J.L. 1993. A genotype of hepatitis D virus that occurs in northern South America. Proc. Natl. Acad. Sci. 90: 9016-9020.

Cenci, C., Barzotti, R., Galeano, F., Corbelli, S., Rota, R., Massimi, L., Di Rocco, C., O'Connell, M.A., and Gallo, A. 2008. Downregulation of RNA editing in pediatric astrocytomas: ADAR2 editing activity inhibits cell migration and proliferation. J. Biol. Chem. 283: 7251-7260.

Chadalavada, D.M., Cerrone-Szakal, A.L., and Bevilacqua, P.C. 2007. Wild-type is the optimal sequence of the HDV ribozyme under cotranscriptional conditions. RNA 13: 2189-2201.

Chang, F.L., Chen, P.J., Tu, S.J., Wang, C.J., and Chen, D.S. 1991. The large form of hepatitis delta antigen is crucial for assembly of hepatitis delta virus. Proc. Natl. Acad. Sci. 88: 8490-8494.

Chao, M., Hsieh, S.Y., Luo, G.X., and Taylor, J. 1991. The antigen of human hepatitis delta virus: The significance of the two major electrophoretic forms. Prog. Clin. Biol. Res. 364: 275-281.

Cheng, Q., Jayan, G.C., and Casey, J.L. 2003. Differential inhibition of RNA editing in hepatitis delta virus genotype III by the short and long forms of hepatitis delta antigen. J. Virol. 77: 7786-7795.

Dawson, T.R., Sansam, C.L., and Emeson, R.B. 2004. Structure and sequence determinants required for the RNA editing of ADAR2 substrates. J. Biol. Chem. 279: 4941-4951.

Diegelman-Parente, A. and Bevilacqua, P.C. 2002. A mechanistic framework for co-transcriptional folding of the HDV genomic ribozyme in the presence of downstream sequence. J. Mol. Biol. 324: $1-16$.

Gandy, S.Z., Linnstaedt, S.D., Muralidhar, S., Cashman, K.A., Rosenthal, L.J., and Casey, J.L. 2007. RNA editing of the human herpesvirus 8 kaposin transcript eliminates its transforming activity and is induced during lytic replication. J. Virol. 81: 13544-13551.

Gee, A.H., Kasprzak, W., and Shapiro, B.A. 2006. Structural differentiation of the HIV-1 polyA signals. J. Biomol. Struct. Dyn. 23: $417-428$.

Jayan, G.C. and Casey, J.L. 2002a. Increased RNA editing and inhibition of hepatitis delta virus replication by high-level expression of ADAR1 and ADAR2. J. Virol. 76: 3819-3827.

Jayan, G.C. and Casey, J.L. 2002b. Inhibition of hepatitis delta virus RNA editing by short inhibitory RNA-mediated knockdown of ADAR1 but not ADAR2 expression. J. Virol. 76: 12399-12404.

Jayan, G.C. and Casey, J.L. 2005. Effects of conserved RNA secondary structures on hepatitis delta virus genotype I RNA editing, replication, and virus production. J. Virol. 79: 11187-11193.

Kasprzak, W. and Shapiro, B. 1999. Stem Trace: An interactive visual tool for comparative RNA structure analysis. Bioinformatics 15: $16-31$.

Kasprzak, W., Bindewald, E., and Shapiro, B.A. 2005. Structural polymorphism of the HIV-1 leader region explored by computational methods. Nucleic Acids Res. 33: 7151-7163.

Keegan, L.P., Leroy, A., Sproul, D., and O'Connell, M.A. 2004. Adenosine deaminases acting on RNA (ADARs): RNA-editing enzymes. Genome Biol. 5: 209.

Kuo, M.Y., Chao, M., and Taylor, J. 1989. Initiation of replication of the human hepatitis delta virus genome from cloned DNA: Role of delta antigen. J. Virol. 63: 1945-1950.

Lehmann, K.A. and Bass, B.L. 1999. The importance of internal loops within RNA substrates of ADAR1. J. Mol. Biol. 291: 1-13.

Linnstaedt, S.D., Kasprzak, W.K., Shapiro, B.A., and Casey, J.L. 2006. The role of a metastable RNA secondary structure in hepatitis delta virus genotype III RNA editing. RNA 12: 1521-1533.
Maas, S., Kawahara, Y., Tamburro, K.M., and Nishikura, K. 2006. A-to-I RNA editing and human disease. RNA Biol. 3: 1-9.

Manock, S.R., Kelley, P.M., Hyams, K.C., Douce, R., Smalligan, R.D., Watts, D.M., Sharp, T.W., Casey, J.L., Gerin, J.L., Engle, R., et al. 2000. An outbreak of fulminant hepatitis delta in the Waorani, an indigenous people of the Amazon basin of Ecuador. Am. J. Trop. Med. Hyg. 63: 209-213.

Mathews, D.H., Sabina, J., Zuker, M., and Turner, D.H. 1999. Expanded sequence dependence of thermodynamic parameters improves prediction of RNA secondary structure. J. Mol. Biol. 288: 911-940.

Niro, G.A., Smedile, A., Andriulli, A., Rizzetto, M., Gerin, J.L., and Casey, J.L. 1997. The predominance of hepatitis delta virus genotype I among chronically infected Italian patients. Hepatology 25: 728-734.

Ohman, M., Kallman, A.M., and Bass, B.L. 2000. In vitro analysis of the binding of ADAR2 to the pre-mRNA encoding the GluR-B R/ G site. RNA 6: 687-697.

Polson, A.G. and Bass, B.L. 1994. Preferential selection of adenosines for modification by double-stranded RNA adenosine deaminase. EMBO J. 13: 5701-5711.

Polson, A.G., Bass, B.L., and Casey, J.L. 1996. RNA editing of hepatitis delta virus antigenome by dsRNA-adenosine deaminase. Nature 380: 454-456.

Polson, A.G., Ley III, H.L., Bass, B.L., and Casey, J.L. 1998. Hepatitis delta virus RNA editing is highly specific for the amber/W site and is suppressed by hepatitis delta antigen. Mol. Cell. Biol. 18: 19191926.

Repsilber, D., Wiese, S., Rachen, M., Schroder, A.W., Riesner, D., and Steger, G. 1999. Formation of metastable RNA structures by sequential folding during transcription: Time-resolved structural analysis of potato spindle tuber viroid (-)-stranded RNA by temperature-gradient gel electrophoresis. RNA 5: 574-584.

Riedmann, E.M., Schopoff, S., Hartner, J.C., and Jantsch, M.F. 2008. Specificity of ADAR-mediated RNA editing in newly identified targets. RNA 14: 1110-1118.

Sato, S., Cornillez-Ty, C., and Lazinski, D.W. 2004. By inhibiting replication, the large hepatitis delta antigen can indirectly regulate amber/W editing and its own expression. J. Virol. 78: 8120-8134.

Shapiro, B.A. and Kasprzak, W. 1996. STRUCTURELAB: A heterogeneous bioinformatics system for RNA structure analysis. J. Mol. Graph. 14: 194-205, 222-224.

Shapiro, B.A. and Navetta, J. 1994. A massively parallel genetic algorithm for RNA secondary structure prediction. J. Supercomput. 8: 195-207.

Shapiro, B.A. and Wu, J.C. 1996. An annealing mutation operator in the genetic algorithms for RNA folding. Comput. Appl. Biosci. 12: $171-180$.

Shapiro, B.A., Bengali, D., Kasprzak, W., and Wu, J.C. 2001a. RNA folding pathway functional intermediates: Their prediction and analysis. J. Mol. Biol. 312: 27-44.

Shapiro, B.A., Wu, J.C., Bengali, D., and Potts, M.J. 2001b. The massively parallel genetic algorithm for RNA folding: MIMD implementation and population variation. Bioinformatics 17: 137-148.

Shapiro, B.A., Kasprzak, W., Grunewald, C., and Aman, J. 2006. Graphical exploratory data analysis of RNA secondary structure dynamics predicted by the massively parallel genetic algorithm. J. Mol. Graph. Model. 25: 514-531.

Tucker, B.J. and Breaker, R.R. 2005. Riboswitches as versatile gene control elements. Curr. Opin. Struct. Biol. 15: 342-348.

Wang, K.S., Choo, Q.L., Weiner, A.J., Ou, J.H., Najarian, R.C., Thayer, R.M., Mullenbach, G.T., Denniston, K.J., Gerin, J.L., and Houghton, M. 1986. Structure, sequence, and expression of the hepatitis delta viral genome. Nature 323: 508-514.

Wong, S.K., Sato, S., and Lazinski, D.W. 2003. Elevated activity of the large form of ADAR1 in vivo: Very efficient RNA editing occurs in the cytoplasm. RNA 9: 586-598. 

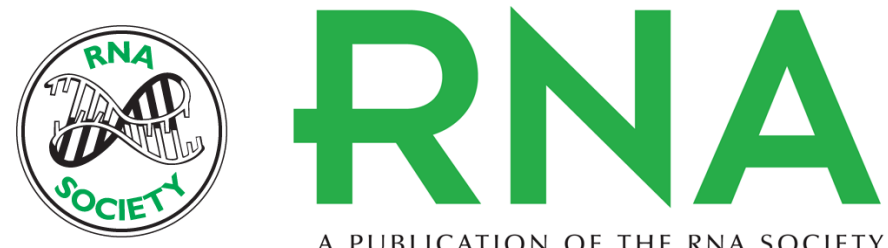

A PUBLICATION OF THE RNA SOCIETY

\section{The fraction of RNA that folds into the correct branched secondary structure determines hepatitis delta virus type 3 RNA editing levels}

Sarah D. Linnstaedt, Wojciech K. Kasprzak, Bruce A. Shapiro, et al.

RNA 2009 15: 1177-1187 originally published online April 21, 2009

Access the most recent version at doi:10.1261/rna.1504009

References This article cites 47 articles, 24 of which can be accessed free at:

http://rnajournal.cshlp.org/content/15/6/1177.full.html\#ref-list-1

License

Email Alerting Receive free email alerts when new articles cite this article - sign up in the box at the Service top right corner of the article or click here. 